\title{
Coexpression and Transcriptome analyses identify active Apomixis-related genes in Paspalum notatum leaves
}

Fernanda A. de Oliveira', Bianca B. Z. Vigna ${ }^{2}$, Carla C. da Silva ${ }^{1}$, Alessandra P. Fávero², Frederico P. de Matta ${ }^{2}$, Ana L. S. Azevedo ${ }^{3}$ and Anete P. de Souza ${ }^{1,4^{*}}$ (D)

\begin{abstract}
Background: Paspalum notatum exhibits both sexual and apomictic cytotypes and, thus, is considered a good model for studies of apomixis because it facilitates comparative approaches. In this work, transcriptome sequencing was used to compare contrasting P. notatum cytotypes to identify differential expression patterns and candidate genes involved in the regulation of expression of this trait.

Results: We built a comprehensive transcriptome using leaf and inflorescence from apomictic tetraploids and sexual diploids/tetraploids and a coexpression network based on pairwise correlations between transcript expression profiles. We identified genes exclusively expressed in each cytotype and genes differentially expressed between pairs of cytotypes. Gene Ontology enrichment analyses were performed to better interpret the data. We de novo assembled 114,306 reference transcripts. In total, 536 candidate genes possibly associated with apomixis were detected through statistical analyses of the differential expression data, and several interacting genes potentially linked to the apomixis-controlling region, genes that have already been reported in the literature, and their neighbors were transcriptionally related in the coexpression network.

Conclusions: Apomixis is a highly desirable trait in modern agriculture due to the maintenance of the characteristics of the mother plant in the progeny. The reference transcriptome, candidate genes and their coexpression network identified in this work represent rich resources for future grass breeding programs.
\end{abstract}

Keywords: Differential expression, Gene coexpression network, Apomixis, RNA sequencing, Paspalum

\section{Background}

RNA sequencing (RNA-seq) is the most effective method for simultaneously predicting new transcripts and identifying differentially expressed genes among distinct tissues, genotypes, abiotic conditions and developmental stages [1]. Conversely, considering the large amount of data generated from RNA-seq, new approaches that efficiently extract meaningful associations from highly multivariate datasets are needed [2]. Transcriptome coexpression studies can show how complex phenotypes depend on the activity of coordinated

\footnotetext{
* Correspondence: anete@unicamp.br

${ }^{1}$ Centro de Biologia Molecular e Engenharia Genética (CBMEG), Universidade Estadual de Campinas (UNICAMP), Campinas, SP, Brazil

${ }^{4}$ Departamento de Biologia Vegetal, Instituto de Biologia, UNICAMP,

Campinas, SP, Brazil

Full list of author information is available at the end of the article
}

batteries of genes [3]. Therefore, the construction of coexpression networks based on gene expression data using correlation metrics provides valuable information regarding alterations in biological systems in response to differential gene expression patterns [2, 4].

The center of origin for the Paspalum genus is tropical South America, but centers of diversity have been recognized in the Brazilian cerrados and the campos of Argentina, Uruguay and Southern Brazil [5]. P. notatum Flüggé, also known as bahiagrass, exhibits numerous characteristics that make it an interesting system for studies investigating apomixes [6]. P. notatum is considered an agamic complex that includes different ploidy levels and reproductive modes, among which diploids $(2 \mathrm{n}=2 \times=20)$ are sexual and polyploids $(3 \times, 4 \times, 5 \times$, and $6 \times)$ are pseudogamous apomicts [7]; no $4 \times$ sexual 
cytotypes have been found in nature [8]. Apomixis is a mode of asexual reproduction through seeds in which plant offspring are genetically identical to the female parent [9]. The inheritance of apomixis is controlled by a single complex dominant locus, i.e., epigenetically controlled parthenogenesis [10], with the capacity to form endosperm with a ratio of 4:1 (maternal:paternal) [6]. The apomixis-controlling region (ACR) in Paspalum is smaller than those of other apomictic systems [11] and does not demonstrate recombination, and a relatively narrow region linked to apomixis is conserved among distinct species $[12,13]$. Comparative mapping of the ACR has shown synteny with a portion of the long arm of chromosome 12 in rice in P. simplex, $P$. malacophyllum and $P$. procurrens [12-14]. In $P$. notatum, chromosomal rearrangements have been reported in regions corresponding to rice chromosomes 2 and 12 [12, 15-17].

Apomixis can be classified in the following two ways: sporophytic, which occurs when the embryo develops from an ovule's somatic cell through numerous mitotic divisions, and gametophytic, in which a chromosomally unreduced embryo sac can be formed from either the megaspore mother cell (diplospory) or a nearby nucellar cell (apospory) without fertilization (parthenogenesis) through a process termed apomeiosis. Endosperm formation is required for the production of viable seeds [18]. Apomixis is widely distributed among angiosperms, among which Poaceae represents the family with the largest number of apomictic genera, with the 47 apomictic species of Paspalum standing out in particular $[6,19]$. Due to the polyploid nature of apomicts, genetic and genomic analyses are challenging.

Polyploidization is known to cause immediate and extensive genomic changes, including sequence rearrangements and/or elimination, changes in DNA methylation and loss of balance in gene expression [11]. Apomixis is frequently correlated with polyploidy and might have arisen through deregulation of the sexual developmental pathway due to an increase in the number of genomic complements through a mechanism regulated by genetic and epigenetic components [20,21].

Apomixis has potential significance for agriculture by allowing the maintenance of heterosis in hybrid progeny. An understanding of the genomic structure of the apomictic locus is likely a prerequisite for the manipulation of the sexual pathway in model plants or economically important crops. The discovery of key genes associated with the components of apomixis and the isolation of sequence candidates have already been reported in Paspalum [18, 20, 22-34]. However, insight into the genetic mechanisms underlying asexual reproduction in natural apomicts is still needed for the development of a stable and universal system applicable to breeding programs [32]. In addition, given the complexity of this trait, an understanding of the genomic structure of the apomictic locus is likely to be an essential prerequisite for manipulation of the sexual pathway in model plants or economically important crops [35]. Nevertheless, the suppression of recombination events around the ACR limits direct genetic strategies to isolate the mechanism triggering apospory [25]. In this context, reverse genetics approaches have garnered extreme interest in the study of apomixis to identify and validate genes that are differentially expressed in apomicts and to conduct more meticulous investigations aiming to detect their positions and functions [25,33].

The objective of this study was to use RNA-seq technology to obtain a transcriptome and comprehensively analyze the gene expression profiles of leaves and florets from apomictic and sexual genotypes with different ploidy levels in $P$. notatum. Our study revealed differentially expressed genes among the genotypes, and through the presentation of a genetic correlation network obtained by examining the de novo transcriptome, we investigate the main biological processes of genes potentially linked to the ACR. Inferences regarding candidate genes involved in the regulation of the expression of apomixis in $P$. notatum are also discussed. These candidate genes may be used to further explore and clarify the mechanisms regulating apomixis in forage grasses.

\section{Results \\ DNA content and mode of reproduction of $P$. notatum accessions}

The cytoembryological analysis (Additional file 1: Figure S1) confirmed the expected mode of reproduction of the accessions based on the literature (Tables 1 and 2). The 2C DNA content of all plants from accessions BGP_22 and BGP_306 was half the 2C DNA content from all plants from BGP_30, BGP_34, BGP_115, and BGP_216. Therefore, all evaluated plants had DNA contents compatible with the described ploidy levels of their respective accessions in the literature (Tables 1 and 2).

\section{RNA-seq analysis and de novo transcriptome assembly}

In the present study, $49.0 \mathrm{~Gb}$ of data were obtained, of which $87 \%$ of the reads sequenced had a Phred quality score $>30$. Floret and leaf tissues were used to assemble a $P$. notatum reference transcriptome based on 313,198 , 097 high-quality 72-bp paired-end reads. In total, 203, 808 transcripts were assembled, including a set of 114 , 306 nonredundant transcripts (56.08\% of all transcripts) (Additional file 2: Table S1), with an average length of $750.51 \mathrm{bp}$, an N50 of $906 \mathrm{bp}$ and a GC percentage of $47.60 \%$. According to the length distribution of the nonredundant transcripts, $21,585(18.88 \%)$ transcripts were longer than $1 \mathrm{~kb}$, a size range that commonly confers a high annotation rate. More than half of all annotated 
Table 1 Paspalum notatum accessions evaluated in this study

\begin{tabular}{lllll}
\hline BGP code $^{\text {a }}$ & Former code & Origin & Collector $^{\text {b }}$ & Chromosome number $^{2}$ \\
\hline 306 & BRA-024236 & Candói (PR), Brazil & VLiGu 14,829 & $2 n=2 \times=20[36]$ \\
22 & BRA-006173 & Bagé (RS), Brazil & VGnMaBd 9607 & $2 n=2 \times=20[37]$ \\
216 & BRA-019470 & Corrientes, Argentina & Q 3664 & $2 n=4 \times=40[38]$ \\
30 & BRA-006467 & Alegrete (RS), Brazil & VMrFrLw 9747 & $2 n=4 \times=40[39]$ \\
34 & BRA-006513 & Uruguaiana (RS), Brazil & VMrFrLw9782 & $2 n=4 \times=40[39]$ \\
115 & BRA-010006 & Laguna (SC), Brazil & VDBdSv 10,137 & $2 n=4 \times=40[39]$
\end{tabular}

BGP: internal code of germplasm bank of Paspalum

${ }^{b}$ Collectors: Bd, I. I. Boldrini; D, M. Dall'Agnol; Fr, J. M. O. Freitas; Gn, J. O. N. Gonçalves; Gu, A. Guglieri; Li, L. Essi; Lw, H. M. Longhi-Wagner; Ma, M. C. Assis; Mr., C. O. C. Moraes; Q, C. L. Quarín; Sv, G. P. da Silva; V, J. F. M. Valls; LAT, latitude (decimal degrees); LONG, longitude (decimal degrees)

transcripts were $>500 \mathrm{bp}$ in length (Additional file 3 : Figure S2).

Bowtie aligner mapped $94.39 \%$ of the sequenced reads onto the assembled transcripts, of which $88.79 \%$ were concordantly mapped paired ends. Considering the nonredundant transcript set, $73.78 \%$ of the sequenced reads were mapped $(66.65 \%$ paired reads aligned concordantly). Of these reads, 82.97 and $83.48 \%$ of the sequencing reads belonged to sexual and apomictic samples, respectively, showing similar representation within the transcriptome. The BUSCO analysis included 956 conserved single-copy plant orthologs; the transcriptome showed high assembly completeness with 664 (69\%) complete sequences (532 as a single copy and 132 as multiple copies) and 169 (17\%) fragmented sequences. One hundred and twenty-three (12\%) BUSCO plant orthologs were not identified in the reference $P$. notatum transcriptome.

\section{Transcriptome annotation}

Among the 114,306 transcripts, 51,939 (45.44\%) were similar to proteins from the NCBI Nr, 34,750 (30.40\%) were similar to proteins from UniProtKB/Swiss-Prot, and 54,568 (47.74\%) were similar to proteins from Phytozome. Overall, 21,537 (41.47\%) transcripts were assigned to 4614 GO terms in the following categories: 2510 biological process, 1486 molecular function, and 618 cellular components. KEGG annotation was possible for 9221 transcripts belonging to 129 pathways, and the most represented was the Purine Metabolism Pathway (1079 members), followed by Metabolism of Thiamine (907 members) and Starch and Sucrose Metabolism (336 members). Overall, 33,873 transcripts remained without hits after searching against all protein databases; among these transcripts, open reading frames (ORFs) were found in 15,107 transcripts, and 2347 transcripts were complete. The sequences that showed ORFs without annotation require further investigation since these sequences may represent genes that have not yet been described and possibly new genes unique to $P$. notatum.

\section{Expression levels of genes and identification of DEGs}

A comparison of the transcript expression levels of the sequenced samples between the two tissues revealed genes that were expressed in only the genotypes of a phenotypic class (Fig. 1). These transcripts were considered "specific" to that class. Thus, 19,304 specific transcripts were found exclusively in the $4 \mathrm{X}$ apo group, 2173 specific transcripts were found exclusively in the $2 \mathrm{X}$ sex group, and 217 specific transcripts were found exclusively in the $4 \mathrm{X}$ sex group. There were no specific transcripts expressed in the florets, as all transcripts present were also expressed in the leaves (Fig. 1).

In the differential gene expression analyses, we considered only the leaf samples that contained the representative transcripts from the transcriptome assembly and examined three biological replicates (clones) per genotype. However, within the same phenotypic class, different expression patterns were observed among the genotypes, mainly in the $4 \mathrm{X}$ apo samples (Additional file 4: Figure S3). Thus, to reduce the effects of the genotype-based differences in expression, we performed a two-step pipeline. First, we selected transcripts that were evenly expressed within the phenotypic classes. A total of 72,318 transcripts were equally expressed among all $4 \mathrm{X}$ apo genotypes, and 47,069 transcripts were equally expressed in the $2 \mathrm{X}$ sex genotypes. This initial analysis was not necessary for $4 \mathrm{X}$ sex because only one genotype was analyzed. From these transcripts, we selected 28,969 transcripts that were expressed in all three phenotypic classes. Second, the pairwise comparisons of the 28,969 transcripts enabled the identification of 2072 DEGs between $2 \mathrm{X}$ sex and 4X apo, including 772 and 1173 overexpressed transcripts, respectively. There were 1173 DEGs between $2 \mathrm{X}$ sex and $4 \mathrm{X}$ sex, including 308 and 798 overexpressed transcripts, respectively, and 1317 genes were identified as DEGs between $4 \mathrm{X}$ apo and 4X sex, including 618 and 661 overexpressed transcripts, respectively. Subsequently, we inferred the enriched functions of the identified DEGs that 
Table 2 DNA content and modes of reproduction of the bahiagrass accessions evaluated in this study

\begin{tabular}{|c|c|c|c|c|c|c|c|c|c|}
\hline \multirow{2}{*}{$\begin{array}{l}\text { Accession } \\
\text { (BGP code) }\end{array}$} & \multicolumn{4}{|l|}{ DNA content } & \multicolumn{5}{|c|}{ Mode of reproduction } \\
\hline & Plants & $\begin{array}{l}2 \mathrm{C} \\
(\mathrm{pg})\end{array}$ & $\begin{array}{l}\Delta 2 \mathrm{C} \text { among } \\
\text { plants }\end{array}$ & $\begin{array}{l}\text { Accession } \\
\text { mean 2C (pg) }\end{array}$ & $\begin{array}{l}\% \text { meiotic } \\
\text { embryo } \\
\text { sacs }\end{array}$ & $\begin{array}{l}\% \text { aposporic } \\
\text { embryo } \\
\text { sacs }\end{array}$ & $\begin{array}{l}\text { \% sterile } \\
\text { embryo } \\
\text { sacs }\end{array}$ & $\begin{array}{l}\text { Number of } \\
\text { ovaries analyzed }\end{array}$ & Classification \\
\hline \multirow[t]{5}{*}{30} & BGP30_1 ${ }^{a}$ & 3,2 & 0,4 & 3,2 & 38 & 56 & 6 & 100 & Facultative apomixis \\
\hline & BGP30_2 $2^{a}$ & 3,1 & & & & & & & \\
\hline & BGP30_3 & 2,9 & & & & & & & \\
\hline & BGP30_4 & 2,8 & & & & & & & \\
\hline & BGP30_5 $5^{\text {ac }}$, & 3 & & & & & & & \\
\hline \multirow[t]{5}{*}{34} & BGP34_1 ${ }^{a}, c_{1} c$ & 2,9 & 0,5 & 2,8 & 20 & 80 & 0 & 100 & Facultative apomixis \\
\hline & BGP34_2 & 2,9 & & & & & & & \\
\hline & BGP34_3 $3^{\mathrm{a}}$ & 2,5 & & & & & & & \\
\hline & BGP34_4 & 2,7 & & & & & & & \\
\hline & BGP34_5 ${ }^{a}$ & 3 & & & & & & & \\
\hline \multirow[t]{5}{*}{115} & BGP115_1 & 2,7 & 0,3 & 2,68 & 0 & 95 & 5 & 100 & Obligate apomixis \\
\hline & BGP115_2 $2^{\mathrm{ac}}$ & 2,9 & & & & & & & \\
\hline & BGP115_3 $3^{\mathrm{a}}$ & 2,6 & & & & & & & \\
\hline & BGP115_4 & 2,6 & & & & & & & \\
\hline & BGP115_5 $5^{\mathrm{a}}$ & 2,7 & & & & & & & \\
\hline \multirow[t]{5}{*}{216} & BGP216_1 ${ }^{\mathrm{a}}$, & 2,6 & 0,6 & 2,82 & 100 & 0 & 0 & 300 & Sexual \\
\hline & BGP216_2 $2^{\mathrm{a}}$ & 2,7 & & & & & & & \\
\hline & BGP216_3 & 2,9 & & & & & & & \\
\hline & BGP216_4 & 3,2 & & & & & & & \\
\hline & BGP216_5 $5^{\mathrm{a}}$ & 2,7 & & & & & & & \\
\hline \multirow[t]{5}{*}{22} & BGP22_1 & 1,5 & 0,3 & 1,52 & 100 & 0 & 0 & 300 & Sexual \\
\hline & BGP22_2 & 1,7 & & & & & & & \\
\hline & BGP22_3 $3^{a b c}$, & 1,4 & & & & & & & \\
\hline & BGP22_4 $4^{a}$ & 1,4 & & & & & & & \\
\hline & BGP22_5 $5^{\mathrm{a}}$ & 1,5 & & & & & & & \\
\hline \multirow[t]{5}{*}{306} & BGP306_1 ${ }^{\mathrm{ac}}$, & 1,5 & 0,1 & 1,44 & 100 & 0 & 0 & 300 & Sexual \\
\hline & BGP306_2 & 1,5 & & & & & & & \\
\hline & BGP306_3 & 1,4 & & & & & & & \\
\hline & BGP306_4 $4^{\mathrm{a}}$ & 1,4 & & & & & & & \\
\hline & BGP306_5 $5^{\mathrm{a}}$ & 1,4 & & & & & & & \\
\hline
\end{tabular}

andicates plants with foliar RNA evaluated

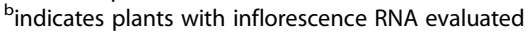

cindicates plants with DNA evaluated

were over- or underexpressed (Additional file 5: Figure S4, Additional file 6: Figure S5 and Additional file 7: Figure S6).

Among the identified DEGs, we isolated the same 510 transcripts that were differentially expressed between the $4 \mathrm{X}$ apomictic and the 2X sexual samples and between the $4 \mathrm{X}$ apomictic and $4 \mathrm{X}$ sexual samples, regardless of the ploidy of the sexual samples. The GO terms enriched in this set belonged to 26 biological processes (BP), 13 molecular functions (MF), and three cellular components (CC) (Fig. 2). We considered these transcripts to be potentially involved in the determination of the reproductive mode.

\section{qRT-PCR validation of RNA-seq data}

We validated the in silico analyzes using a subset of the DEGs between the apomictic and sexual samples through an independent technique, i.e., qRT-PCR. Out of the eight genes selected for internal control, three showed nonspecific DNA amplification and were discarded, and five were successfully amplified, showed desirable expression stability and coefficients of variance 


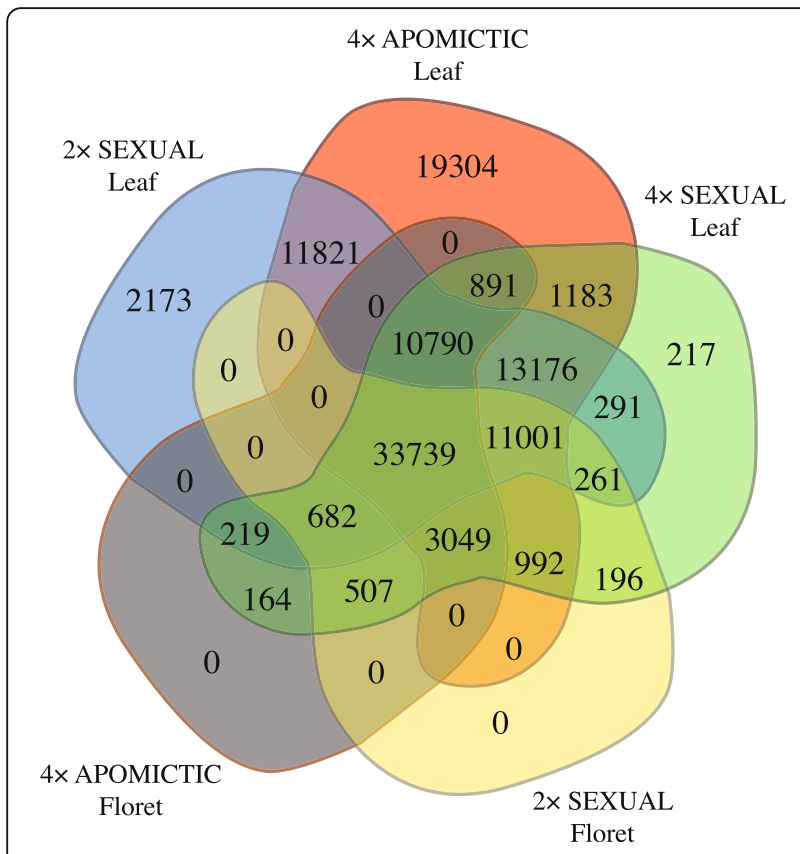

Fig. 1 Venn diagram showing the distribution of the P. notatum transcripts. Expression level of transcripts from two tissues (floret and leaf) from each phenotypic class (sexual diploid, sexual tetraploid, and apomictic tetraploid) among the samples, showing $\mathrm{M}$ values $<0.5$ and $\mathrm{CV}$ values $<0.25$ (Additional file 8: Table S2). Of these genes, one gene was selected as an internal control for qRT-PCR verification of the $2 \mathrm{X}$ sex vs. $4 \mathrm{X}$ apo analyses (01RefGen-Pnot), and one gene was selected for verification of the $4 \mathrm{X}$ apo vs. $4 \mathrm{X}$ sex analyses (04RefGen-Pnot) due to their expression stability between the samples of each group. Different internal controls were used for the different groups of samples because we opted for increased accuracy in the analyses instead of risking a biased result using the same control for the sample groups. The transcriptionally stable genes we adopted in our qRT-PCR may also serve as reference genes for other studies.

The 18 primer pairs designed for the target DEGs, were initially evaluated through PCR using genomic DNA as a template. Eight did not amplify or presented nonspecific amplification and were discarded. Two primer pairs were not evaluated in qRT-PCR but showed interesting results in terms of DNA amplification. The first was designed from a transcript that was exclusively expressed in apomict samples in RNA-seq analyses (i.e., showing a zero expression value in the sexual samples). This transcript did not amplify using genomic DNA from sexual samples as PCR templates. For the second primer pair, although genomic DNA amplification occurred in all samples, when complementary DNA (cDNA) was used as a PCR template, only the apomictic

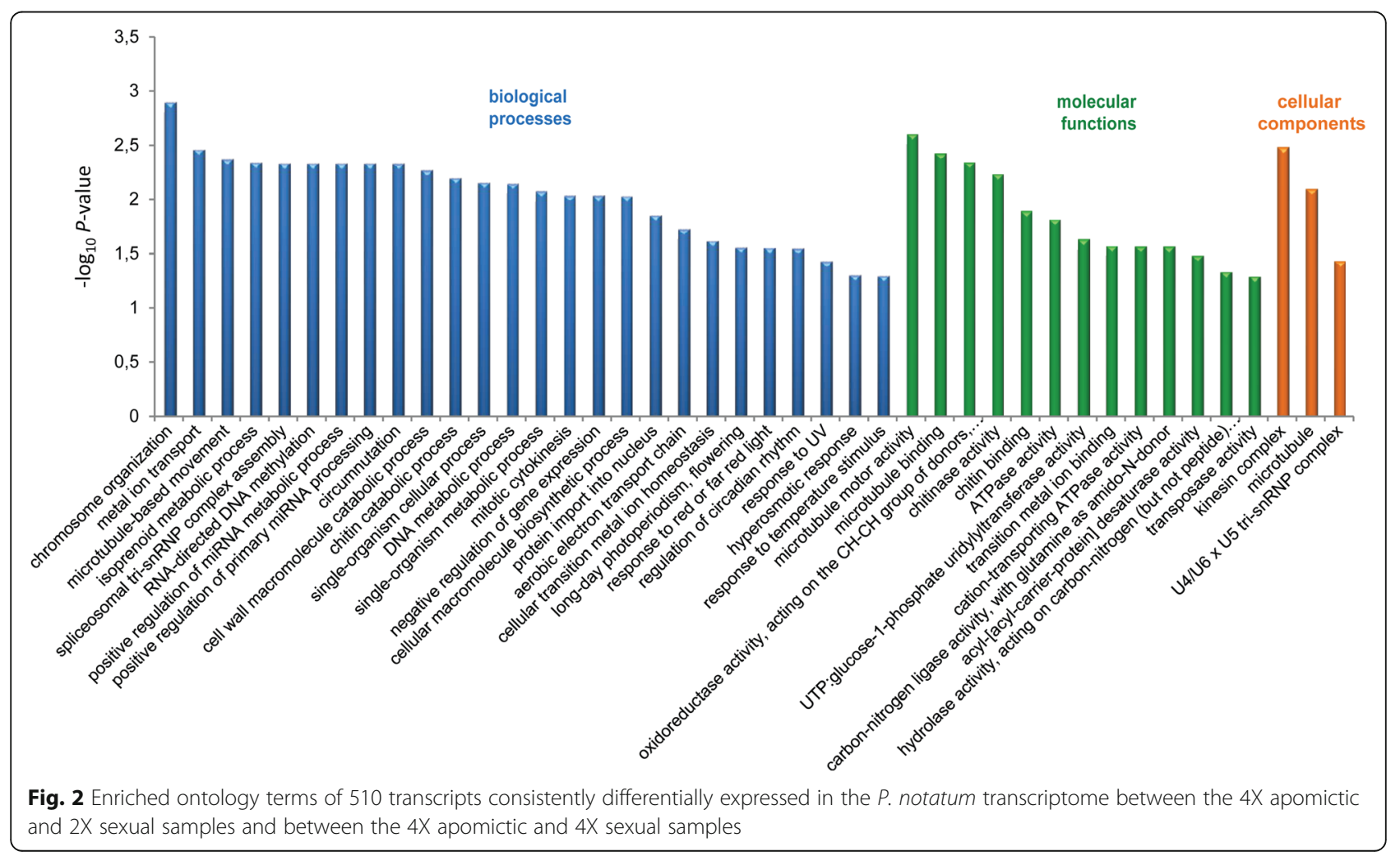


samples presented amplicons. The expression level of this transcript estimated from RNA-seq of the same samples was very low in the $2 \mathrm{X}$ sex samples. These results suggest that the first transcript, which is expressed only in apomictic cytotypes, is exclusive to the genomes of these samples, whereas the second transcript was poorly expressed or silenced in sexual samples.

Finally, eight primer pairs successfully produced amplicons and were used for qRT-PCR validation (Additional file 9: Table S3), revealing results that were consistent with those obtained through RNA-seq (Additional file 10: Figure S7 and Additional file 11: Figure S8).

\section{Detailed search for potential apomixis genes}

We detected 1612 transcripts in the P. notatum reference transcriptome that showed high similarity to the putative ACR region syntenic to rice chromosomes 2 and 12. Of these transcripts, 1356 transcripts aligned to the correspondent rice chromosome 2, and 256 transcripts aligned to rice chromosome 12 . Interestingly, 40 transcripts were a part of the set identified as apomictic specific; nine transcripts were specific to the $2 \mathrm{X}$ sex samples, and six transcripts were specific to the $4 \mathrm{X}$ sex samples. Moreover, considering the analyses of the DEGs, 16 putative ACR transcripts were differentially expressed between $2 \mathrm{X}$ sex and $4 \mathrm{X}$ apo; 20 DEGs were observed between $4 \mathrm{X}$ apo and $4 \mathrm{X}$ sex; and 17 DEGs were observed between $2 \mathrm{X}$ sex and $4 \mathrm{X}$ sex. However, in total, there were 89 different transcripts, because some were repeated as differentially expressed in more than one pairwise comparison.

These findings, especially this 89-transcript set (Additional file 12: Table S4), represent valuable genes that deserve to be carefully investigated to determine their roles in apomixis and whether they are effective within the ACR in $P$. notatum.

The BLAST results shown in Additional file 13: Table S5 highlight the similarity scores of the $P$. notatum transcripts to Paspalum sequences previously associated with apomixis, revealing that our transcriptome contains genes already found to be involved in asexual reproductive development.

\section{Transcript coexpression network}

We identified 879,481 connections among the 53,262 transcripts from the RNA-seq data (Fig. 3). The transcripts

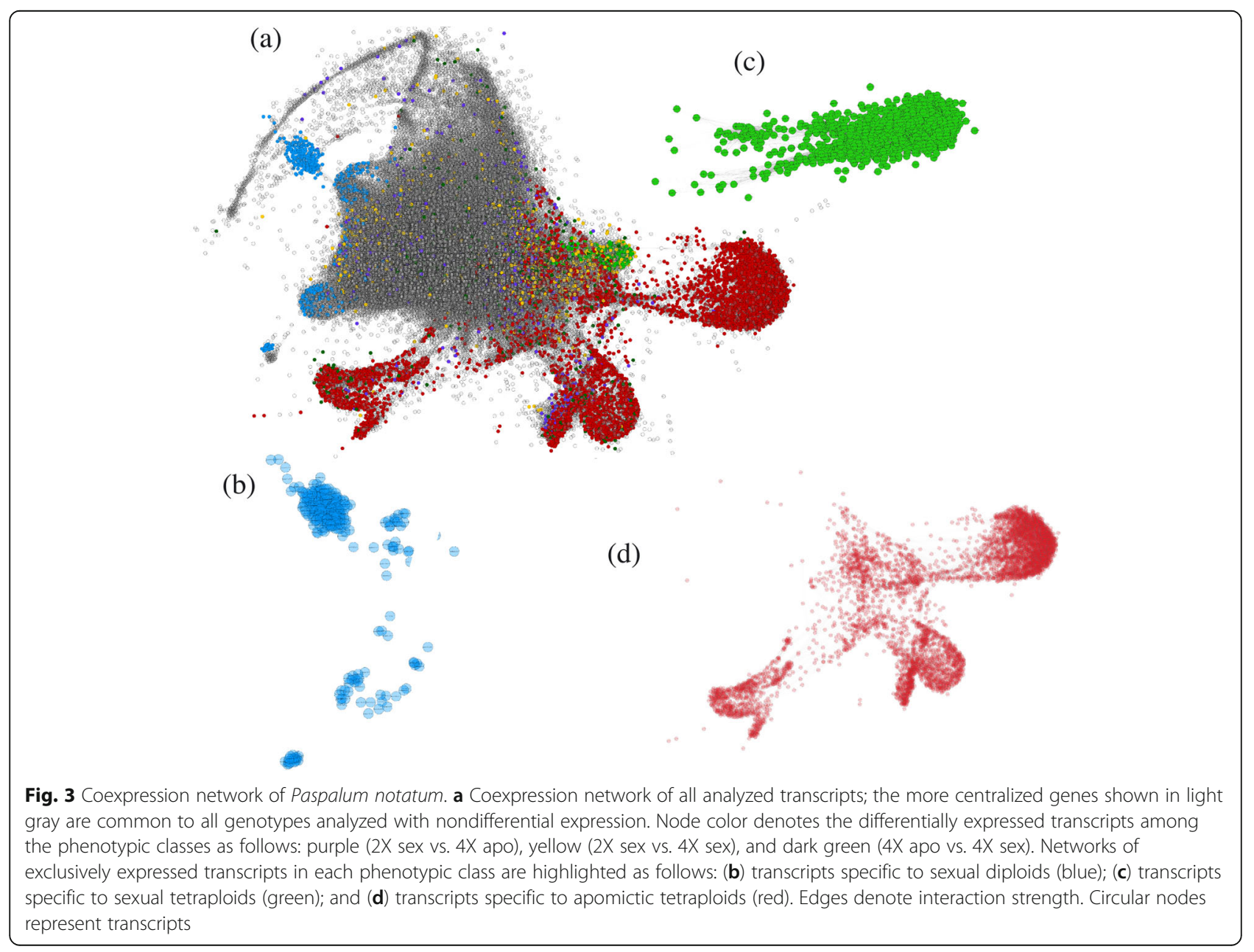


were grouped into 642 clusters according to their correlated pattern of expression level. In the network, the specific transcripts in each phenotypic class tended to form coexpression modules (Fig. 3bd). Despite this subdivision, the relevant biological relationships of these transcripts with all remaining transcripts can be recovered in a fully integrated network.

To identify the direct correlations among the 89 differentially expressed and/or specific putative ACR genes and their first neighbors, we retrieved a subnetwork composed of 536 strongly correlated transcripts (Fig. 4). We used a GO enrichment analysis to summarize the main functions of this transcript set, which included $43 \mathrm{BP}, 31 \mathrm{MF}$ and $17 \mathrm{CC}$ (Fig. 5).

\section{Discussion}

We constructed a $P$. notatum reference transcriptome using RNA-seq to investigate the gene expression changes in apomictic and sexual genotypes. This analysis enabled the assembly of a nonredundant transcriptome containing 114,306 transcripts from florets and leaves from six distinct genotypes with different ploidy levels and reproductive modes. Robust metrics indicated that the transcriptome was a quality assembly with a high degree of integrity.

Although we did not sequence inflorescence samples at different developmental stages to search for candidate apomixis-linked genes, we isolated expression patterns of phenotypic classes, aiming both at removing transcripts whose expression could be related to the effect of genotype and at identifying transcripts expressed in all

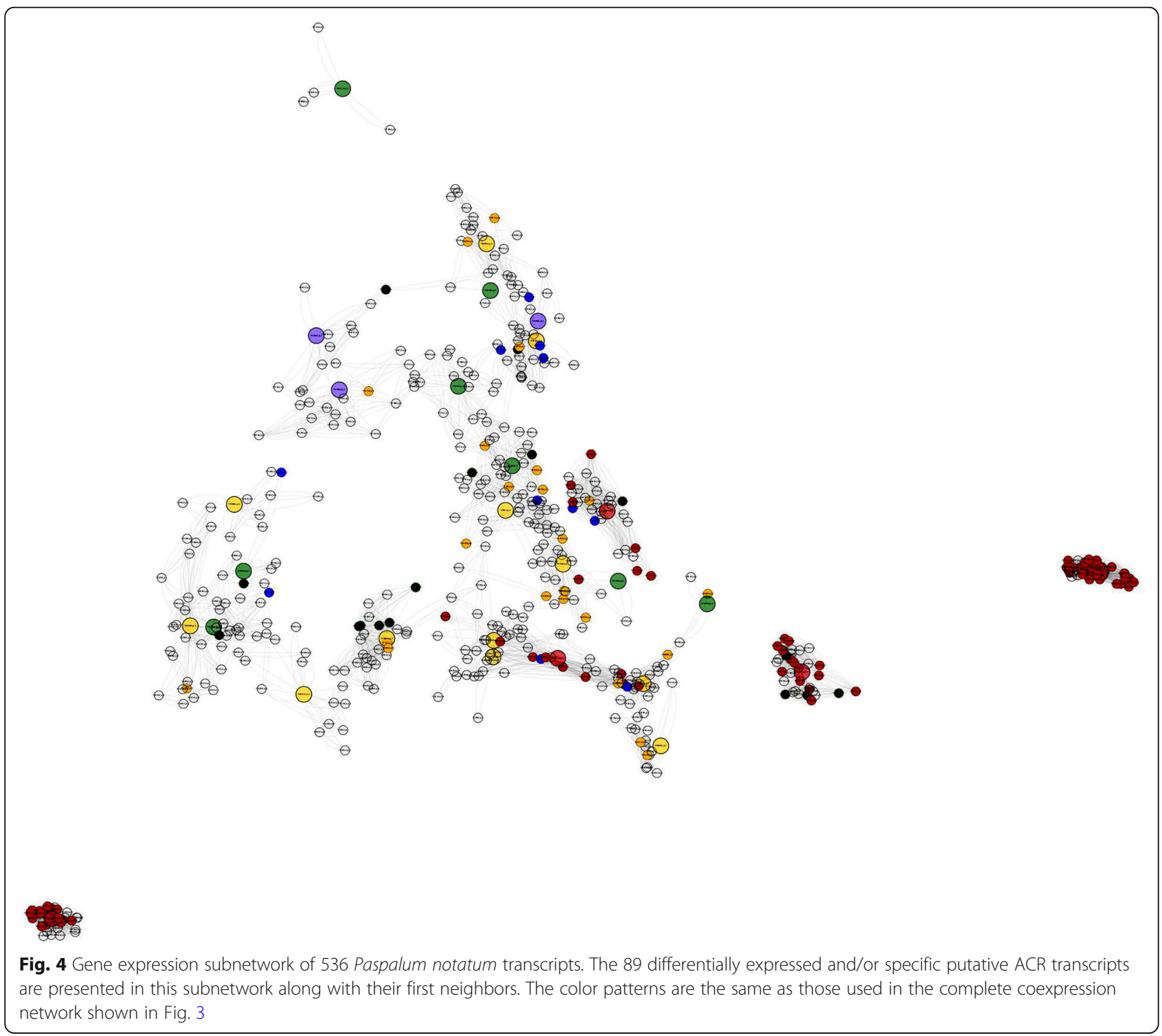




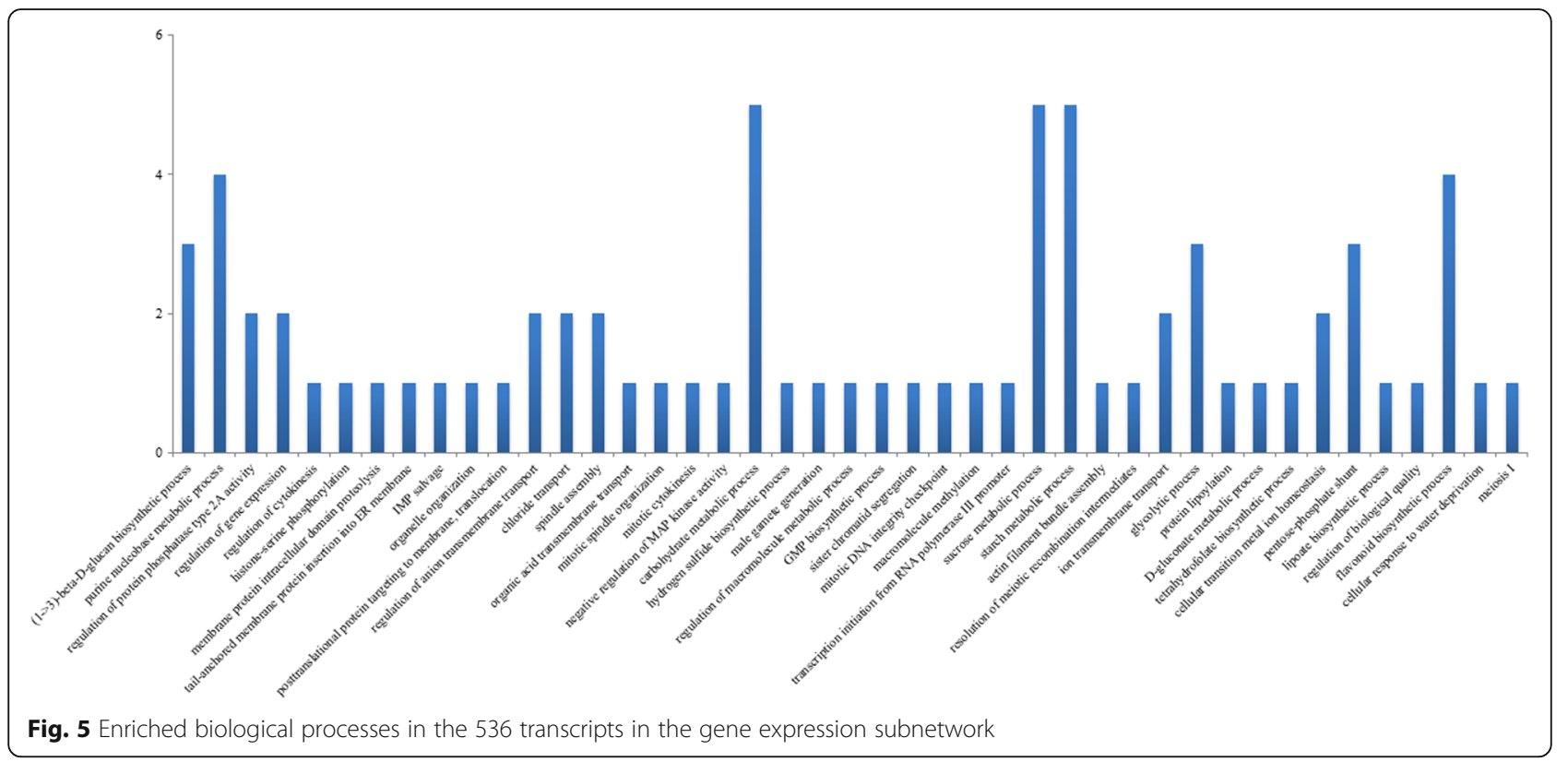

genotypes within the same phenotypic class. Differential expression analyses of leaf samples from sexual and apomictic cytotypes allowed us to identify DEGs that may be representative of reproductive mode and not dependent on ploidy level.

The GO classification of the assembled transcriptome into 4614 known terms was used to perform functional enrichment analyses. Based on the enriched GO terms of a set of 510 DEGs detected between apomictic and sexual, we emphasize "positive regulation of miRNA metabolic process (GO:2000630)" and "regulation of primary miRNA processing (GO:2000636)", both of which activate or increase the frequency, rate, or extent of miRNA production. Furthermore, miRNA is directly related to "DNA metabolic process (GO:0006259)", which decreases the rate of gene expression (negative regulation of gene expression - GO:0010629) through an epigenetic RNA-based gene silencing process (RNA-directed DNA methylation - GO:0080188). Five transcripts were mainly involved in these processes and showed higher expression levels in the apomict samples; these transcripts correspond to a pseudoARR-B transcription factor; pre-mRNA splicing factor, putative (Ricinus communis); hypothetical protein SORBIDRAFT_05g016770; uncharacterized protein, LOC100501330 (Zea mays); and an uncharacterized protein LOC103641690. The significant enrichment of terms associated with the regulation of gene expression by epigenetic silencing among the transcripts was higher in the apomictic samples compared with that in the sexual samples of bahiagrass. This result is consistent with the growing body of evidence that suggests that apomixis arises from the deregulation of the sexual pathway, where epigenetic mechanisms play a significant role in at least some elements of apomictic development $[6,40]$.
These 510 DEGs along with the 19,304 transcripts that were exclusively expressed in the apomictic samples represent a set of candidate genes that deserves further investigation. In apospory, gene expression occurs at specific stages, such as apomeiosis, parthenogenesis, and endosperm development. The increased or decreased expression of some genes during these stages may hinder the analysis of differential expression between sexual and apomictic genotypes [33, 41]. Nonetheless, we believe that the identification of DEGs as performed here allowed for the detection of gene expression patterns related to characteristics shared among genotypes independently of physiological or developmental particularities. Thus, using selected transcripts with a detectable expression pattern among all genotypes of each phenotypic class may have minimized that influence. Simultaneously, our approach increased the potential to discover candidate genes involved in many steps of the apomictic process. It is important to emphasize that the group of apomictic genotypes consisted of two facultative apomictic genotypes and one obligatory apomictic genotype and that all selected expressed genes were expressed in the obligatory apomictic sample. However, some amount of background noise might be present. Thus, future reverse genetic experiments based on qRT-PCR and in situ hybridization could be useful for identifying the role of specific genes throughout the apomixis process.

The size of the ACR in Paspalum is smaller than those in other apomictic systems [6], but the lack of recombination hinders the use of map-based cloning approaches in studies investigating this region [25]. Thus, the discovery of candidate genes that are potentially located within this region is extremely valuable for an understanding of the 
complex regulatory network of gene-gene interactions. Thus, DEGs could be used for the future manipulation of the trait, which has outstanding importance in agricultural biotechnology.

Furthermore, the recovery of the previously published sequences demonstrates the representative and informative nature of the assembled transcriptomes and enhances our understanding of global RNA expression. In Paspalum, the first attempt to understand the molecular basis of apomixis was performed by Pessino et al. [23]. This study led to the identification of three small sequences expressed during early megagametophyte development in apomictic plants of $P$. notatum. Here, we recovered the reported sequences as a single representative transcript (Additional file 13: Table S5) that is similar to a kinesin motor protein involved in microtubule-based movement. This protein has been reported to be differentially expressed between apomictic and sexual plants [22, $25,42]$, and the same pattern was observed in the transcripts assembled in this work.

Subsequently, several other candidate genes have been reported using $P$. notatum and $P$. simplex [20, 23-25], and the first study using RNA-seq was performed in bahiagrass based on expression profiling of apomictic and sexual flowers [33]. Some candidate genes have been investigated in more detail [28, 30-32]. However, many questions remain unanswered, and further research is needed to define the relationships among the structure, position, and function of known apomixis-linked genes [6].

Based on a set of genes selected through differential expression patterns, we recovered previously published sequences in the $P$. notatum transcriptome. n20gap-1, which is one of these previously reported genes in the literature, is a Lorelei-like $P$. notatum gene $[25,28]$ that encodes a GPI-anchored protein that supposedly plays a role in the final stages of the apomixis developmental cascade. Laspina et al. [25] reported that this sequence is linked to the chromosomal locus governing apospory at a genetic distance of $22 \mathrm{cM}$. Here, one transcript similar to n20gap-1 was present in the set of transcripts potentially integrated within the ACR, corresponding to rice chromosome 2. Additionally, we found differentially expressed transcripts that aligned with the sequences characterized by Podio et al. [30], corresponding to Somatic Embryogenesis Receptor-Like Kinase (SERK), in addition to numerous other transcripts with the same annotation. The candidate SERK-like genes play crucial roles in somatic embryogenesis in angiosperms and have been reported to be related to apomixis. We also identified $P$. notatum transcripts that showed similarity to the PnTgs1-like gene that encodes a trimethylguanosine synthase-like protein, which plays a fundamental role in nucellar cell fate, and its diminished expression has been reported to correlate with the initiation of the apomictic pathway [31]. Additionally, two transcripts were similar to the sequence of PsORC3, which seems to play an active role in the mechanisms repressing sexuality in apomictic $P$. simplex [32].

Functionally related genes tend to be transcriptionally coordinated [43]. Therefore, the construction of a coexpression network provided a powerful resource for the identification of transcripts that are coregulated with specific genes and DEGs despite the lack of detectable differences in expression among the samples. The subnetwork containing 536 coexpressed transcripts possibly associated with the expression of apomixis, is an example of a valuable approach to finding likely biological relationships among genes of interest using transcriptomic approaches. By starting on a smaller scale of transcripts that are DEGs and/or specific genes possibly integrated into the ACR and combining information by adding their nearest neighbor genes, we obtained a broader view of the processes involved in the regulation of candidate genes. The significantly enriched BP, such as "male gamete generation (GO: 0048232)", "sister chromatid segregation (GO: 0000819)", "meiosis I (GO: 0007127)", "resolution of meiotic recombination intermediates (GO: 0000712)", "regulation of cytokinesis (GO: 0032465)", "mitotic cytokinesis (GO: 0000281)", "spindle assembly (GO: 0051225)", "mitotic spindle organization (GO: 0007052)", and "mitotic DNA integrity checkpoint (GO: 0044774)", were associated with plant reproduction. Additionally, the subnetwork was enriched in epigenetic processes, such as "macromolecule methylation (GO: 0043414)", "histone-serine phosphorylation (GO: 0035404)", "regulation of protein phosphatase type 2A activity (GO: 0034047)", and "negative regulation of MAP kinase activity (GO: 0043407)". The transcripts in the subnetwork were associated with reproductive processes and the regulation of epigenetic changes by modulating histones. It is important to note that the MAP kinase activity was negatively regulated in this work, and this gene family also has been reported as being related to the apomictic reproduction. The mitogen-activated protein 3-kinase (MAP3K) gene family has been identified as differentially expressed between apomictic and sexual flowers of $P$. notatum [25] and flowers of P. simplex [20] and might play a role in the parthenogenetic development of embryos in both species [6]. A gene essential for the formation of unreduced embryo sacs in $P$. notatum was identified from the characterization of MAP3K retrieved from transcriptomic surveys [44]. The information presented in the gene coexpression network in this study may highlight candidate genes for the study of apomixis in this species.

In this study, we applied RNA-seq technology and bioinformatics methods to assemble a useful transcriptome and identify differences between apomictic and sexual 
reproduction. Our results reveal DEGs and genes exclusively expressed in apomicts or sexuals that are potentially associated with the ACR genomic region, including the apo locus. The gene coexpression network developed by this work represents an advanced and promising genetic technique for studies in P. notatum. In this work, the technique enabled a more comprehensive analysis of the transcripts obtained from our P. notatum transcriptome that are potentially involved in the regulation of apomixis. The validation of genes in this set of candidates may provide valuable insight to enhance our understanding of apospory. Moreover, these genes may be considered in screening for molecular markers linked to apomixis in $P$. notatum, which will be crucial for boosting breeding programs for apomictic forage grasses.

\section{Methods}

\section{Plant materials and RNA extraction}

Six accessions of $P$. notatum belonging to the Germplasm Bank of Paspalum and maintained by Embrapa Pecuária Sudeste, São Carlos, SP, Brazil $\left(22^{\circ} 01^{\prime} \mathrm{S}\right.$ and $47^{\circ} 54^{\prime} \mathrm{W}$; $856 \mathrm{~m}$ above sea level) (Table 1) were chosen based on their origins (different ecotypes), genetic dissimilarity [45], ploidy levels and reproduction modes. The Q3664 (BGP 216) accession is a hybrid from a cross between a sexual colchicine-induced tetraploid (PT-2) and a white-stigma apomictic tetraploid and is characterized as a facultative apomictic accession with a high level of sexuality (>70\%) [46].

Five clones of each accession were planted in $8 \mathrm{~L}$ pots, filled with 1:1 soil/vermiculite and grown in a greenhouse under identical environmental conditions. The climate is humid subtropical (according to the KöppenGeiger classification system), with annual average low and high temperatures of 15.3 and $27.0^{\circ} \mathrm{C}$ and a total rainfall of $1422.8 \mathrm{~mm}$ occurring mainly during the spring and summer seasons [47]. Young leaf samples from three clones of each accession were collected during the summer (December). One clone of the accessions BGP34 (apomictic tetraploid) and BGP22 (sexual diploid) presented inflorescences, which were collected. All leaf and floret samples were immediately placed in liquid nitrogen and subsequently stored at $-80^{\circ} \mathrm{C}$ until RNA extraction. The total RNA was isolated according to Oliveira et al. [48]. RNA integrity was assessed in a $1 \%$ agarose denaturing gel and quantified using a NanoVue Plus spectrophotometer (GE Healthcare Life Sciences, Little Chalfont, UK).

\section{Mode of reproduction through cytoembryological analysis}

The mode of reproduction of the plants was confirmed using the embryo sac clarification method proposed by Young et al. [49], with minor modifications. Inflorescences at anthesis (when the embryo sac is fully developed) were fixed in FAA (95\% ethanol, distilled water, glacial acetic acid, and formalin $40 \%$ at $40: 13: 3: 3 \mathrm{v} / \mathrm{v}$ ) for $24 \mathrm{~h}$ at room temperature. The embryo sacs were clarified as followed: 85\% ethanol; absolute ethanol; ethanol:methyl salicylate (1:1); ethanol:methyl salicylate (1:3); and 100\% methyl salicylate (two times). The samples remained in each solution for $24 \mathrm{~h}$. Then, 100 to 300 embryo sacs per accession were evaluated under an Axiophot microscope (Carl Zeiss, Jena, Germany) using the differential interference contrast (DIC) microscopy technique.

\section{DNA content estimation through flow cytometry}

Flow cytometry analyses were performed to confirm the exact ploidy level (Table 1). Approximately $5 \mathrm{mg}$ of young leaf tissue from each clone were used (Table 2). Pisum sativum cv. Ctirad $(2 \mathrm{C}=9.09 \mathrm{pg})$ was used as an internal control [50]. The samples were triturated in Petri dishes containing $800 \mu \mathrm{L}$ of LB01 buffer $(0.45425 \mathrm{~g}$ TRIS, $0.186125 \mathrm{~g}$ NaEDTA, $0.0435 \mathrm{~g}$-spermine, $0.29225 \mathrm{~g} \mathrm{NaCl}$, $1.491 \mathrm{~g} \mathrm{KCl}$, and $250 \mu \mathrm{L}$ of Triton $\mathrm{X}-100$ in $250 \mathrm{~mL}$ of distilled water, $7.5 \mathrm{pH}, 0.11 \% \mathrm{v} / \mathrm{v} \beta$-mercaptoethanol) [51]. The nuclear suspension was filtered through a $40-\mu \mathrm{m}$ mesh and incubated at room temperature for $5 \mathrm{~min}$, followed by the addition of $25 \mu \mathrm{L}$ of propidium iodide and $25 \mu \mathrm{L}$ of RNase. For each sample, at least 10,000 nuclei were analyzed with a FACSCalibur flow cytometer (Becton Dickinson, New Jersey, USA). Histograms were generated using Cell Quest software and analyzed using Flowing Software (available at http://www.flowingsoftware.com). The nuclear DNA index (pg) was estimated based on the value of the G1 peak. We calculated the mean value of $C$ per clone and accession and the difference between the highest and lowest values $(\Delta)$ observed in the replicates of each accession.

\section{RNA-seq library, Illumina sequencing, and data quality control}

cDNA libraries were constructed from 18 leaf and two floret samples using a TruSeq RNA Sample Preparation Kit (Illumina Inc., San Diego, CA, USA) following the manufacturer's instructions. Library quality was confirmed using the Agilent 2100 Bioanalyzer (Agilent Technologies, Palo Alto, CA, USA) and quantified via quantitative real-time PCR (qPCR) (Illumina protocol SY-930-10-10). Clustering was performed using a TruSeq Paired-End Cluster Kit on a cBot (Illumina Inc.). Paired-end sequencing was performed on an Illumina Genome Analyzer IIx Platform with TruSeq SBS 36Cycle kits (Illumina Inc.) following the manufacturer's specifications. RNA-seq was performed with floret libraries in two separate lanes without biological replicates. The remaining 18 leaf libraries from six different accessions (Table 1), each with three biological 
replicates, were distributed randomly in the flow cell with three libraries per lane.

The raw data was converted to FastQ files containing 72-bp reads. Quality control was performed using the NGS QC Toolkit v2.3.3 [52]. Initially, high-quality reads (Phred quality score $\geq 20$ in at least $75 \%$ of bases) and reads with more than 60 bases were selected. Subsequently, reads were trimmed at the $3^{\prime}$ end for the removal of barcodes.

\section{Transcriptome assembly and completeness assessment}

The reads from the leaves and florets were de novo assembled into a reference transcriptome with Trinity v2.0.2 [53] using default settings. The contig redundancy was minimized through the selection of the first Butterfly sequence from each Chrysalis component, which is considered the most representative contig. The reads were mapped to the transcriptome using Bowtie2 sequence aligner v2.2.5 [54]. The Benchmarking Universal Single-Copy Orthologs (BUSCO) tool, an approach assessing conserved orthologs among plant species in a set of sequences [55], was used to estimate the completeness of the transcriptome assembly.

\section{Transcriptome annotation}

The transcripts were aligned to proteins from the NCBI nonredundant $(\mathrm{Nr})$ database, UniProtKB/Swiss-Prot and Phytozome v9.0 using the BLASTX algorithm with an evalue cutoff of 1e-06. The Gene Ontology (GO) terms were retrieved using Blast2GO software. We used REVIGO [56] to summarize the GO term sets. The transcripts were assigned to metabolic pathways via the Kyoto Encyclopedia of Genes and Genomes (KEGG) database [57].

\section{Transcript expression levels}

The expression levels were estimated using the FPKM method (expected number of fragments per kilobase of transcript sequence per million base pairs sequenced). The read counts were obtained using Bowtie v2-2.1.0 and normalized using the RSEM software (RNA-seq by expectation maximization) [58]. A Venn diagram was created using the online platform available at http://bioinformatics.psb.ugent.be/webtools/Venn/. A principal component analysis (PCA) was used to assess the expression patterns of the sequenced genotypes.

\section{Differential expression analysis}

EBSeq [59] was used to identify the differentially expressed genes (DEGs) using the FPKM values at a false discovery rate $(F D R) \leq 0.05$. Transcripts with a log2-fold change $(\mathrm{FC}) \geq 1.5$ in abundance were regarded as overexpressed. The GO enrichment analysis was carried out using the 'goseq' 1.24.0 Bioconductor package
[60]. The $P$-values were subjected to Bonferroni correction; adjusted $\mathrm{P}$-values $\leq 0.05$ were considered enriched and summarized using REVIGO [56].

The genotypes were grouped according to their phenotypic classes as sexual diploids (2X sex), sexual tetraploids ( $4 \mathrm{X}$ sex) and apomictic tetraploids ( $4 \mathrm{X}$ apo) and compared in pairwise differential expression analyses. A two-step pipeline was used for the identification of the DEGs (Fig. 6). In the first step, the objective was to select a list of equally expressed transcripts among genotypes belonging to the same phenotypic class. This procedure allowed the detection of gene expression patterns related to characteristics that were shared among the genotypes, independently of physiological or developmental particularities. Thus, EBSeq was used to estimate the pairwise posterior probabilities of a transcript being equally expressed between genotypes as follows: $2 \mathrm{X}$ sex (BGP22 and BGP306) and 4X apo (BGP30, BGP34 and BGP115); thus, a list of equally expressed transcripts (PPEE $\geq 0.95$ ) among genotypes belonging to the same phenotypic class were retained for the second step of the analysis. The second step consisted of another round of pairwise differential expression analyses (PPDE) between phenotypic classes: (i) $2 \mathrm{X}$ sex and $4 \mathrm{X}$ apo; (ii) $2 \mathrm{X}$ sex and $4 \mathrm{X}$ sex; (iii) $4 \mathrm{X}$ apo and $4 \mathrm{X}$ sex.

\section{Quantitative reverse transcription PCR (qRT- PCR) validation}

To verify the reliability and accuracy of the transcriptome data and validate the differential expression results, 18 DEGs were randomly selected for quantification through reverse transcription PCR (qRT-PCR). Eight transcripts that displayed similar expression patterns based on FPKM were selected to be evaluated as control genes. The primer sets were designed using Primer3Plus software [61]; the target amplicon size was set to 70 $150 \mathrm{bp}$, with an optimal annealing temperature of $60^{\circ} \mathrm{C}$ and an optimal primer length of $20 \mathrm{bp}$. All primer pairs were initially tested in regular PCRs using genomic DNA from the same samples used in RNA-seq as the template. Only primers that amplified the genomic DNA of all genotypes were used in the following qRT-PCR. Primer pairs that successfully amplified the DNA of all genotypes and showed an amplification efficiency of 90$110 \%$ and $R^{2}>0.99$ were used for the relative expression analyses.

Total RNA (500 ng) was used for cDNA synthesis using the QuantiTect Reverse Transcription Kit (Qiagen Inc., Chatsworth, CA, USA). The cDNAs were diluted $(1: 10)$ in nuclease-free water, and $2 \mu \mathrm{L}$ was used for qRT-PCR. qRT-PCR was performed using a CFX384 Real-Time PCR Detection System with iTaq Universal SYBR Green Supermix (Bio-Rad Laboratories Inc., Hercules, CA, USA) according to the manufacturer's 


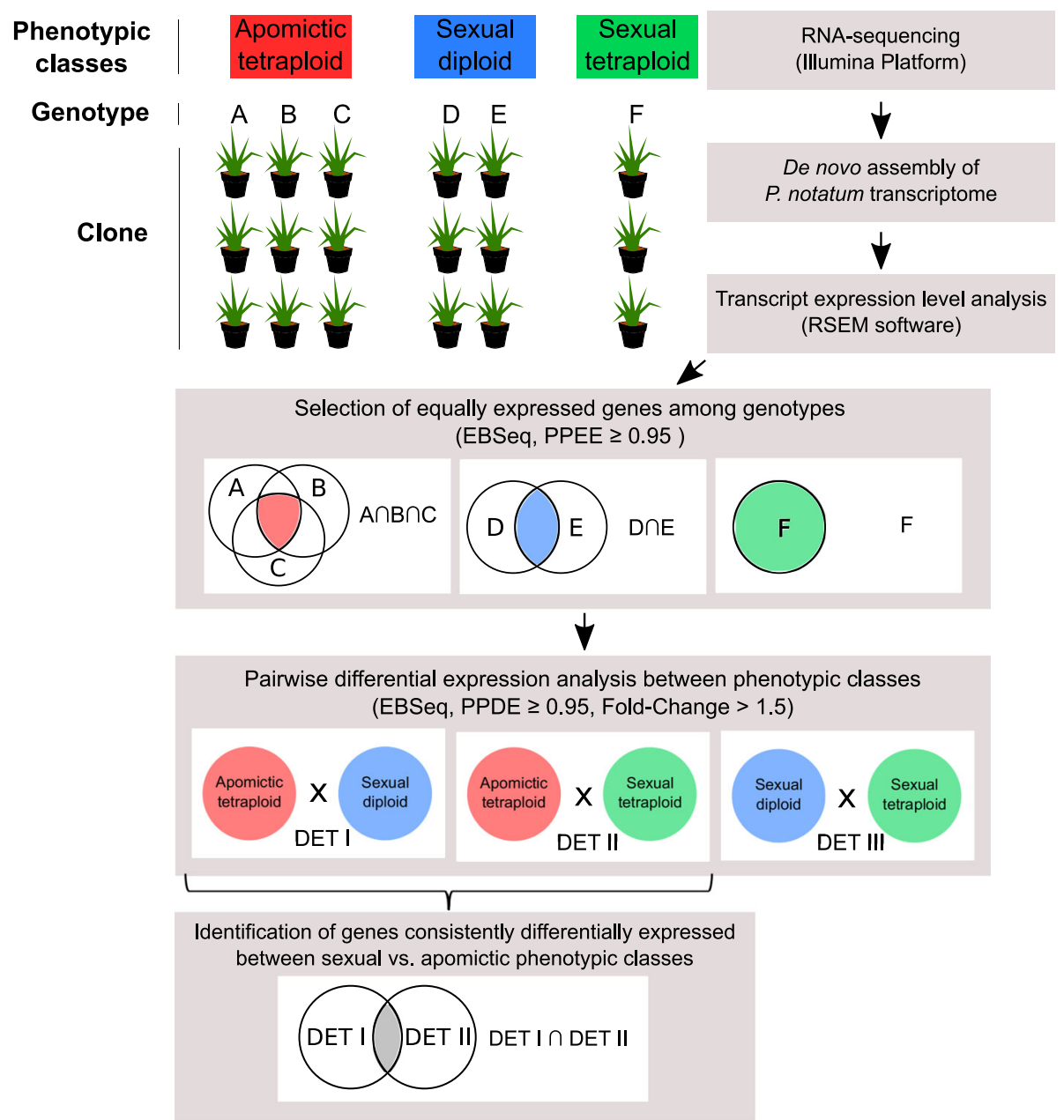

Fig. 6 Workflow of differential expression analyses used in this study. We performed pairwise differential expression analyses of all three phenotypic classes (apomictic tetraploid " $4 X$ apo", sexual diploid " $2 X$ sex" and sexual tetraploid " $4 X$ sex"). Each phenotypic class had different genotypes with three clones. First, the objective was to select a list of equally expressed transcripts among genotypes belonging to the same phenotypic class. EBSeq was used to estimate the pairwise posterior probabilities of a transcript being equally expressed (PPEE) among genotypes of the same phenotypic class as follows: apomictic tetraploid $(A \times B ; A \times C ; B \times C)$ and sexual diploid $(D \times E)$. Transcripts with PPEE $\geq 0.95$ were kept for the second step of the analysis. Second, another round of pairwise differential expression analyses among phenotypic classes was performed as follows: (i) $4 \mathrm{X}$ apo vs. $2 \mathrm{X}$ sex; (ii) $4 \mathrm{X}$ apo vs. $4 \mathrm{X}$ sex; and (iii) $2 \mathrm{X}$ sex vs. $4 \mathrm{X}$ sex. The objective was to select a list of transcripts that presented pairwise posterior probabilities of being differentially expressed (PPDE $\geq 0.95$ ). Additionally, we identified a list of transcripts that were consistently differentially expressed between the sexual and apomictic samples independently of the ploidy level (diploid or tetraploid)

instructions, and the final primer concentration was $0.3 \mu \mathrm{M}$. The reaction conditions were $95^{\circ} \mathrm{C}$ for $10 \mathrm{~min}$, followed by 40 cycles of $95^{\circ} \mathrm{C}$ for $30 \mathrm{~s}$ and $60^{\circ} \mathrm{C}$ for 1 min. No-template controls for each primer pair were included, and all experiments were performed in triplicate using independent samples. The specificity of the amplicons was confirmed through analysis of the melting curve with temperatures increasing from $65^{\circ} \mathrm{C}$ to $95^{\circ} \mathrm{C}$ with increments of $0.5{ }^{\circ} \mathrm{C}$. The baseline and $\mathrm{Cq}$ (quantification cycle) values were automatically determined, and the expression values were estimated using $\Delta \Delta \mathrm{Ct}$ by CFX Manager 2.1 software (Bio-Rad Laboratories, Inc., USA). The reference genes were selected according to the gene expression stability values $(M<0.5)$ and coefficients of variance $(\mathrm{CV}<0.25)$ among the samples. Mann-Whitney U-tests were performed to estimate the statistical significance.

\section{Search for putative apomixis genes}

A comparative analysis of $P$. notatum transcripts with their syntenic counterparts on rice chromosomes conservatively linked to apomixis in Paspalum species was performed [6]; we aligned all $P$. notatum transcripts to rice transcripts using BLASTN in the highlighted area of rice chromosomes 2 and 12, which were delimited by a set of molecular markers completely linked to the apospory locus. This region encompassed the C1069 and C996 markers of rice chromosome 12 and between the C560 and C932 markers 
of chromosome $2[6,12,15-17,62]$. We also compared the transcriptome with sequences reported to be potentially associated with apomixis in Paspalum using BLASTN with an e-value cutoff of 1e-05.

\section{Coregulatory networks}

For the coexpression network, we used the FPKM of all assembled transcripts from the floret and leaf tissues with a Pearson correlation coefficient cutoff of $\geq 0.8$. Transcripts showing null values for most clones were excluded to reduce noise. The highest reciprocal rank (HRR) [63] method with a limit of 30 was used to select the strongest edges. A heuristic cluster chiseling algorithm (HCCA) was applied with three-step node vicinity networks (NVN) [63]. Cytoscape 3.4.0 [64] was used for visualization.

\section{Supplementary information}

Supplementary information accompanies this paper at https://doi.org/10. 1186/s12864-020-6518-z

Additional file 1: Figure S1. Clarified embryo sacs visualized by differential interference contrast (DIC) microscopy from the Paspalum notatum accessions evaluated in this work. (A) Apomictic and (B) sexual BGP 30 embryo sacs. (C) Apomictic and (D) sexual BGP 34 embryo sacs. (E) Apomictic embryo sac from BGP 115. (F), (G) and (H) Sexual embryo sacs from BGP 306, BGP 22 and BGP 216, respectively.

Additional file 2: Table S1. Statistics of the assembled transcriptome of Paspalum notatum.

Additional file 3: Figure S2. Length distribution of the number of nonredundant transcripts successfully annotated. Comparison of all nonredundant transcripts and the number of transcripts annotated in the NCBI nonredundant protein database by size range.

Additional file 4: Figure S3. Principal component analysis (PCA) according to the FPKM values of the $P$. notatum transcriptome. PCA of leaf and floret transcriptomes of all genotypes and clones used in RNA-seq.

Additional file 5: Figure S4. Functional classification of enriched overexpressed DEGs in the $2 X$ sex vs. the $4 X$ apo comparison. Gene Ontology biological process (blue boxes), Gene Ontology cellular component (yellow boxes), and Gene Ontology molecular function (orange boxes). a) Categories enriched in overexpressed transcripts in $2 X$ sex and b) categories enriched in overexpressed transcripts in $4 \mathrm{X}$ apo.

Additional file 6: Figure S5. Functional classification of enriched overexpressed DEGs in the $2 X$ sex vs. $4 X$ sex comparison. Gene Ontology biological process (blue boxes), Gene Ontology cellular component (yellow boxes), and Gene Ontology molecular function (orange boxes). a) Categories enriched in overexpressed transcripts in $2 X$ sex and $b$ ) categories enriched in overexpressed transcripts in $4 \mathrm{X}$ sex.

Additional file 7: Figure S6. Functional classification of enriched overexpressed DEGs in the $4 X$ apo vs. $4 X$ sex comparison. Gene Ontology biological process (blue boxes), Gene Ontology cellular component (yellow boxes), and Gene Ontology molecular function (orange boxes). a) Categories enriched in overexpressed transcripts in 4X apo and b) categories enriched in overexpressed transcripts in $4 \mathrm{X}$ sex.

Additional file 8: Table S2. Primer sequences and amplicons of the candidate reference genes evaluated in this study.

Additional file 9: Table S3. Quantitative RT-PCR primer sequences.

Additional file 10: Figure S7. Histograms of gene expression obtained by $\mathrm{qRT}$-PCR. $\mathrm{qRT}$-PCR validation showing the relative expression patterns of 4 genes differentially expressed between the tetraploid apomictic " $4 \mathrm{X}$ apo " (red box) and diploid sexual " $2 \times$ SEX" (blue box) samples. * $P<0.05$ ${ }^{* *} P<0.01$; ${ }^{* *} P<0.001$; statistically significant differences in gene expression between phenotypic classes compared using the Mann-Whitney Utest.

Additional file 11: Figure S8. Histograms of gene expression obtained by $\mathrm{qRT}$-PCR. qRT-PCR validation showing the relative expression patterns of 4 genes differentially expressed between the tetraploid apomictic " $4 X$ apo " (red box) and tetraploid sexual " $4 \mathrm{X}$ sex" (green box) samples. ${ }^{*} \mathrm{P}<$ $0.05 ;{ }^{* *} \mathrm{P}<0.01 ;{ }^{* *} \mathrm{P}<0.001$; statistically significant differences in gene expression between the phenotypic classes compared using the MannWhitney U-test.

Additional file 12: Table S4. Functional classification of the 89 differentially expressed and/or specific putative apomixis-controlling region (ACR) transcripts.

Additional file 13: Table S5. Results of the BLAST search of sequences of $P$. notatum from the literature against the assembled transcriptome.

\section{Abbreviations}

2X sex: Sexual diploids; 4X apo: Apomictic tetraploids; 4X sex: Sexual tetraploids; ACR: Apomixis-controlling region; BP: Gene ontology biological process; BUSCO: Benchmarking universal single-copy orthologs; CC: Gene ontology cellular component; cDNA: Complementary DNA;

Cq: Quantification cycle; CV: Coefficients of variance; DEG: Differentially expressed gene; DIC: Differential interference contrast; FC: Fold change: FDR: False discovery rate; FPKM: Fragments per kilobase of transcript sequence per million base pairs sequenced; GO: Gene ontology; HCCA: Heuristic cluster chiseling algorithm; HRR: Highest reciprocal rank; KEGG: Kyoto encyclopedia of genes and genomes database; M: Gene expression stability; MAP3K: Mitogen-activated protein 3-kinase; MF: Gene ontology molecular function; Nr: NCBI nonredundant protein database; NVN: Node vicinity networks; ORF: Open reading frame; PCA: Principal component analysis; PPDE: Differential expression analyses; PPEE: Equally expressed transcripts; qPCR: Quantitative real-time PCR; qRT-PCR: Quantitative reverse transcription PCR; RNA-seq: RNA sequencing; RSEM: RNA-Seq by expectation maximization; SERK: Somatic embryogenesis receptor-like kinase; SRA: NCBI short read archive

\section{Acknowledgements}

We are grateful to Maria Augusta C. Horta and Natalia F. Murad for their help in generating the coexpression networks and Mariana V. Cruz for critically reading the manuscript. This manuscript was previously posted to bioRxiv https://www.biorxiv.org/content/10.1101/369280v2.

\section{Authors' contributions}

APS, BBZV and FAO conceived and designed the experiments; FAO performed the experiments and analyzed the data; FAO and CCS performed the $\mathrm{APCR}$ analyses; APF prepared and provided the plant material; FPM performed the cytoembryological analyses; ALSA obtained and analyzed the data for the flow cytometry analyses; APS, BBZV and FAO. wrote the manuscript; and APS. and BBZV coordinated the work. All authors critically revised and approved the manuscript.

\section{Funding}

This work was supported by grants from the Fundação de Amparo à Pesquisa do Estado de São Paulo (FAPESP 2008/52197-4) and Coordenação de Aperfeiçoamento de Pessoal de Nível Superior (CAPES, Computational Biology Program). FAO received a PhD fellowship from FAPESP (2013/204470), CAPES-Computational Biology Program, and CAPES-EMBRAPA Program, and a PD fellowship (2018/18527-9) from FAPESP. CCS received a PD fellowship from Conselho Nacional de Desenvolvimento Científico e Tecnológico (CNPq) and FAPESP (2015/24346-9). APS is the recipient of a research fellowship from CNPq. The funding bodies played no role in the design of the study and collection, analysis, and interpretation of data and in writing the manuscript.

\section{Availability of data and materials}

The datasets generated during the current study are available in the NCBI Short Read Archive (SRA) repository, under accession number SRP150615. This research is registered in the Ministry of the Environment (SisGen) under 
the number AA835F9 for the regulation of the Brazilian law number 13.123/ 2015. Other datasets used and/or analyzed during the current study are available from the corresponding author on reasonable request.

\section{Ethics approval and consent to participate}

Not applicable.

\section{Consent for publication}

Not applicable.

\section{Competing interests}

The authors declare that they have no competing interests.

\section{Author details}

${ }^{1}$ Centro de Biologia Molecular e Engenharia Genética (CBMEG), Universidade

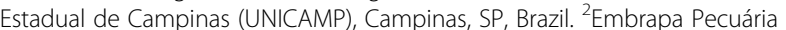
Sudeste, São Carlos, SP, Brazil. ${ }^{3}$ Embrapa Gado de Leite, Juiz de Fora, MG, Brazil. ${ }^{4}$ Departamento de Biologia Vegetal, Instituto de Biologia, UNICAMP, Campinas, SP, Brazil.

Received: 26 June 2019 Accepted: 20 January 2020

Published online: 28 January 2020

\section{References}

1. Huang W, Guo Y, Du W, Zhang X, Li A, Miao X. Global transcriptome analysis identifies differentially expressed genes related to lipid metabolism in Wagyu and Holstein cattle. Sci Rep. 2017;7:5278.

2. Miao X, Luo Q, Zhao H, Qin X. Co-expression analysis and identification of fecundity-related long non-coding RNAs in sheep ovaries. Sci Rep. 2016;6: 39398.

3. Flores-Sandoval E, Romani F, Bowman JL. Co-expression and transcriptome analysis of Marchantia polymorpha transcription factors supports class $C$ ARFs as independent actors of an ancient auxin regulatory module. Front Plant Sci. 2018;9:1345.

4. de la Fuente A. From 'differential expression' to 'differential networking' identification of dysfunctional regulatory networks in diseases. Trends Genet. 2010;26:326-33.

5. Zuloaga FO, Morrone O. Revisión de las especies de Paspalum para América del Sur austral. Ann Mo Bot Garden Monogr Syst Bot. 2005;102:1-297.

6. Ortiz JPA, Quarin CL, Pessino SC, Acuña C, Martínez EJ, Espinoza F, et al. Harnessing apomictic reproduction in grasses: what we have learned from Paspalum. Ann Bot. 2013;112:767-87.

7. Burton GW. The method of reproduction in common Bahia grass, Paspalum notatum. Agron J. 1948;40:443-52.

8. Quarin $\mathrm{CL}$, Urbani MH, Blount AR, Martinez EJ, Hack CM, Burton GW, et al. Registration of Q4188 and Q4205, sexual tetraploid germplasm lines of Bahiagrass. Crop Sci. 2003:43:745-6.

9. Asker S, Jerling L. Apomixis in plants. Boca Raton, FL: CRC Press; 1992.

10. Podio M, Cáceres ME, Samoluk SS, Seijo JG, Pessino SC, Ortiz JPA, et al. A methylation status analysis of the apomixis-specific region in Paspalum spp. suggests an epigenetic control of parthenogenesis. J Exp Bot. 2014;65:6411-24.

11. Adams KL, Wendel JF. Polyploidy and genome evolution in plants. Curr Opin Plant Biol. 2005;8:135-41.

12. Pupilli F, Martinez EJ, Busti A, Calderini O, Quarin CL, Arcioni S. Comparative mapping reveals partial conservation of synteny at the apomixis locus in Paspalum spp. Mol Gen Genomics. 2004;270:539-48.

13. Hojsgaard DH, Martínez EJ, Acuña CA, Quarin CL, Pupilli F. A molecular map of the apomixis-control locus in Paspalum procurrens and its comparative analysis with other species of Paspalum. Theor Appl Genet. 2011;123:959-71.

14. Pupilli F, Labombarda P, Caceres ME, Quarín CL, Arcioni S. The chromosome segment related to apomixis in Paspalum simplex is homoeologous to the telomeric region of the long arm of rice chromosome 12. Mol Breed. 2001; 8:53-61.

15. Martínez EJ, Hopp HE, Stein J, Ortiz JPA, Quarin CL. Genetic characterization of apospory in tetraploid Paspalum notatum based on the identification of linked molecular markers. Mol Breed. 2003:12:319-27.

16. Stein J, Pessino SC, Martínez EJ, Rodriguez MP, Siena LA, Quarin CL, et al. A genetic map of tetraploid Paspalum notatum Flügge (Bahiagrass) based on single-dose molecular markers. Mol Breed. 2007;20:153-66.
17. Podio M, Rodríguez MP, Felitti S, Stein J, Martínez EJ, Siena LA, et al. Sequence characterization, in silico mapping and cytosine methylation analysis of markers linked to apospory in Paspalum notatum. Genet Mol Biol. 2012;35:827-37.

18. Conner JA, Mookkan M, Huo H, Chae K, Ozias-Akins P. A parthenogenesis gene of apomict origin elicits embryo formation from unfertilized eggs in a sexual plant. Proc Natl Acad Sci U S A. 2015;112:11205-10.

19. Hojsgaard D, Klatt S, Baier R, Carman JG, Hörandl E. Taxonomy and biogeography of apomixis in angiosperms and associated biodiversity characteristics. CRC Crit Rev Plant Sci. 2014;33:414-27.

20. Polegri L, Calderini O, Arcioni S, Pupilli F. Specific expression of apomixislinked alleles revealed by comparative transcriptomic analysis of sexual and apomictic Paspalum simplex Morong flowers. J Exp Bot. 2010;61:1869-83.

21. Selva J-P, Pessino SC, Meier MS, Echenique VC. Identification of candidate genes related to polyploidy and/or apomixis in Eragrostis curvula. Am J Plant Sci. 2012;3:403-16.

22. Albertini E, Marconi G, Barcaccia G, Raggi L, Falcinelli M. Isolation of candidate genes for apomixis in Poa pratensis L. Plant Mol Biol. 2004;56: 879-94.

23. Pessino SC, Espinoza F, Martínez EJ, Ortiz JPA, Valle EM, Quarín CL. Isolation of cDNA clones differentially expressed in flowers of apomictic and sexual Paspalum notatum. Hereditas. 2004;134:35-42.

24. Calderini $\mathrm{O}$, Chang SB, Jong H, Busti A, Paolocci F, Arcioni S, et al. Molecular cytogenetics and DNA sequence analysis of an apomixis-linked BAC in Paspalum simplex reveal a non pericentromere location and partial microcolinearity with rice. Theor Appl Genet. 2006;112:1179-91.

25. Laspina NV, Vega T, Seijo JG, González AM, Martelotto LG, Stein J, et al. Gene expression analysis at the onset of aposporous apomixis in Paspalum notatum. Plant Mol Biol. 2008;67:615-28.

26. Yamada-Akiyama H, Akiyama Y, Ebina M, Xu Q, Tsuruta S-I, Yazaki J, et al. Analysis of expressed sequence tags in apomictic guineagrass (Panicum maximum). J Plant Physiol. 2009:166:750-61.

27. Sharbel TF, Voigt ML, Corral JM, Galla G, Kumlehn J, Klukas C, et al. Apomictic and sexual ovules of boechera display heterochronic global gene expression patterns. Plant Cell. 2010;22:655-71.

28. Felitti SA, Seijo JG, González AM, Podio M, Laspina NV, Siena L, et al. Expression of lorelei-like genes in aposporous and sexual Paspalum notatum plants. Plant Mol Biol. 2011;77:337-54.

29. Okada T, Hu Y, Tucker MR, Taylor JM, Johnson SD, Spriggs A, et al. Enlarging cells initiating apomixis in hieracium praealtum transition to an embryo sac program prior to entering mitosis. Plant Physiol. 2013;163:216-31.

30. Podio M, Felitti SA, Siena LA, Delgado L, Mancini M, Seijo JG, et al. Characterization and expression analysis of somatic embryogenesis receptor kinase (SERK) genes in sexual and apomictic Paspalum notatum. Plant Mo Biol. 2014:84:479-95.

31. Siena LA, Ortiz JP, Leblanc O, Pessino S. PnTgs1-like expression during reproductive development supports a role for RNA methyltransferases in the aposporous pathway. BMC Plant Biol. 2014;14:297.

32. Siena LA, Ortiz JPA, Calderini O, Paolocci F, Cáceres ME, Kaushal P, et al. An apomixis-linked ORC3-like pseudogene is associated with silencing of its functional homolog in apomictic Paspalum simplex. J Exp Bot. 2016;67: 1965-78.

33. Ortiz JPA, Revale S, Siena LA, Podio M, Delgado L, Stein J, et al. A reference floral transcriptome of sexual and apomictic Paspalum notatum. BMC Genomics. 2017:18:318.

34. Bocchini M, Galla G, Pupilli F, Bellucci M, Barcaccia G, Ortiz JPA, et al. The vesicle trafficking regulator PN_SCD1 is demethylated and overexpressed in florets of apomictic Paspalum notatum genotypes. Sci Rep. 2018;8:3030.

35. Calderini O, Donnison I, Polegri L, Panara F, Thomas A, Arcioni S, et al. Partial isolation of the genomic region linked with apomixis in Paspalum simplex. Mol Breed. 2011;28:265-76.

36. Dahmer N, Schifino-Wittmann MT, Dall'Agnol M, Castro BD. Cytogenetic data for Paspalum notatum Flügge accessions. Sci Agric. 2008:65:381-8.

37. Pagliarini MS, Carraro LR, Freitas PM, Adamowski EV, Batista LAR, Valls JFM. Cytogenetic characterization of Brazilian Paspalum accessions. Hereditas. 2004;135:27-34.

38. Forbes I, Burton GW. Cytology of diploids, natural and induced tetraploids, and intra-species hybrids of Bahiagrass, Paspalum notatum Flugge. Crop Sci. 1961;1:402-6.

39. Pozzobon MT, Valls JFM. Chromosome number in germplasm accessions of Paspalum notatum (Gramineae). Braz J Genet. 1997;20:29-34. 
40. Brukhin V. Molecular and genetic regulation of apomixis. Russ J Genet. 2017; 53:943-64.

41. Schmidt A, Schmid MW, Klostermeier UC, Qi W, Guthörl D, Sailer C, et al. Apomictic and sexual germline development differ with respect to cell cycle, transcriptional, hormonal and epigenetic regulation. PLoS Genet. 2014;10:e1004476.

42. Albertini E, Marconi G, Reale L, Barcaccia G, Porceddu A, Ferranti F, et al. SERK and APOSTART. Candidate genes for apomixis in Poa pratensis. Plant Physiol. 2005;138:2185-99.

43. Stuart JM, Segal E, Koller D, Kim SK. A gene-coexpression network for global discovery of conserved genetic modules. Science. 2003;302:249-55.

44. Mancini M, Permingeat H, Colono C, Siena L, Pupilli F, Azzaro C, et al. The MAP3K-coding QUI-GON JINN (QGJ) gene is essential to the formation of unreduced embryo sacs in Paspalum. Front Plant Sci. 2018;9:1547.

45. Cidade FW, Vigna BBZ, de Souza FHD, Valls JFM, Dall'Agnol M, Zucchi MI, et al. Genetic variation in polyploid forage grass: assessing the molecular genetic variability in the Paspalum genus. BMC Genet. 2013;14:50.

46. Quarin CL, Burson BL, Burton GW. Cytology of intra- and interspecific hybrids between two cytotypes of Paspalum notatum and P. cromyorrhizon. Bot Gaz. 1984;145:420-6.

47. CEPAGRI/UNICAMP. Centro de Pesquisas Meteorológicas e Climáticas Aplicadas à Agricultura - CEPAGRI/UNICAMP. 2010. https://www.cpa. unicamp.br/o-cepagri. Accessed 14 Nov 2017.

48. Oliveira RR, Viana AJC, Reátegui ACE, Vincentz MGA. Short communication an efficient method for simultaneous extraction of high-quality RNA and DNA from various plant tissues. Genet Mol Biol. 2015;14:18828-38.

49. Young BA, Sherwood RT, Bashaw EC. Cleared-pistil and thick-sectioning techniques for detecting aposporous apomixis in grasses. Can J Bot. 1979;57:1668-72

50. Greilhuber J, Temsch EM, Loureiro JCM. Nuclear DNA content measurements. In: Dolezel J, Greilhuber J, Suda J, editors. Flow cytometry with plant cells: analysis of genes, chromosomes and genomes. Weinheim: Wiley-VCH Verlag; 2007. p. 67-101.

51. Dolezel J. Flow cytometry, its application and potential for plant breeding. In: Lelley T, editor. Current topics in plant cytogenetics related to plant improvement. Vienna: WUV-Universitätsverlag; 1997. p. 80-90.

52. Patel RK, Jain M. NGS QC toolkit: a toolkit for quality control of next generation sequencing data. PLoS One. 2012;7:e30619.

53. Grabherr MG, Haas BJ, Yassour M, Levin JZ, Thompson DA, Amit I, et al. Fulllength transcriptome assembly from RNA-Seq data without a reference genome. Nat Biotechnol. 2011;29:644-52.

54. Langmead B, Trapnell C, Pop M, Salzberg SL. Ultrafast and memory-efficient alignment of short DNA sequences to the human genome. Genome Biol. 2009;10:R25.

55. Simão FA, Waterhouse RM, loannidis $P$, Kriventseva EV, Zdobnov EM BUSCO: assessing genome assembly and annotation completeness with single-copy orthologs. Bioinformatics. 2015;31:3210-2.

56. Supek F, Bošnjak M, Škunca N, Šmuc T. REVIGO summarizes and visualizes long lists of gene ontology terms. PLoS One. 2011;6:e21800.

57. Kanehisa M. KEGG: Kyoto encyclopedia of genes and genomes. Nucleic Acids Res. 2000;28:27-30

58. Li B, Dewey CN. RSEM: accurate transcript quantification from RNA-Seq data with or without a reference genome. BMC Bioinformatics. 2011;12:323.

59. Leng N, Dawson JA, Thomson JA, Ruotti V, Rissman Al, Smits BMG, et al. EBSeq: an empirical Bayes hierarchical model for inference in RNA-seq experiments. Bioinformatics. 2013;29:1035-43.

60. Young MD, Wakefield MJ, Smyth GK, Oshlack A. Gene ontology analysis for RNA-seq: accounting for selection bias. Genome Biol. 2010;11:R14.

61. Untergasser A, Nijveen H, Rao X, Bisseling T, Geurts R, Leunissen JAM. Primer3Plus, an enhanced web interface to primer3. Nucleic Acids Res. 2007;35:W71-4.

62. Labombarda P, Busti A, Caceres ME, Pupilli F, Arcioni S. An AFLP marker tightly linked to apomixis reveals hemizygosity in a portion of the apomixiscontrolling locus in Paspalum simplex. Genome. 2002;45:513-9.

63. Mutwil M, Usadel B, Schutte M, Loraine A, Ebenhoh O, Persson S. Assembly of an interactive correlation network for the arabidopsis genome using a novel heuristic clustering algorithm. Plant Physiol. 2009;152:29-43.

64. Shannon P. Cytoscape: a software environment for integrated models of biomolecular interaction networks. Genome Res. 2003;13:2498-504.

\section{Publisher's Note}

Springer Nature remains neutral with regard to jurisdictional claims in published maps and institutional affiliations.

Ready to submit your research? Choose BMC and benefit from:

- fast, convenient online submission

- thorough peer review by experienced researchers in your field

- rapid publication on acceptance

- support for research data, including large and complex data types

- gold Open Access which fosters wider collaboration and increased citations

- maximum visibility for your research: over $100 \mathrm{M}$ website views per year

At BMC, research is always in progress.

Learn more biomedcentral.com/submissions 